HEMODILUIÇÃO NORMOVOLÊMICA COM SOLUÇÃO HIPERTÔNICA A 7,5\%. PARÂMETROS HEMODINÂMICOS, EFEITOS METABÓLICOS E REPERCUSSÕES LABORATORIAIS

\author{
Liana Maria Tôrres de Araújo
}

\author{
Ribeirão Preto - SP \\ 2008
}




\title{
HEMODILUIÇÃO NORMOVOLÊMICA COM SOLUÇÃO HIPERTÔNICA A 7,5\%. PARÂMETROS HEMODINÂMICOS, EFEITOS METABÓLICOS E REPERCUSSÕES LABORATORIAIS
}

\author{
Tese de Doutorado apresentada à \\ Faculdade de Medicina de Ribeirão Preto \\ para obtenção do título de Doutor em \\ Ciências Médicas. Área de concentração: \\ Ortopedia, Traumatologia e Reabilitação. \\ Opção: Anestesiologia \\ Orientador: Prof. Dr. Luis Vicente Garcia
}




\section{FOLHA DE APROVAÇÃO}

Liana Maria Tôrres de Araújo

Hemodiluição normovolêmica com solução hipertônica a 7,5\%. Parâmetros hemodinâmicos, efeitos metabólicos e repercussões laboratoriais.

Tese de Doutorado apresentada à Faculdade de Medicina de Ribeirão Preto para obtenção do título de Doutor em Ciências Médicas. Área de concentração: Ortopedia, Traumatologia e Reabilitação. Opção: Anestesiologia

Orientador: Prof. Dr. Luis Vicente Garcia

Aprovada em:

Banca examinadora

Prof. Dr.

Instituição: Assinatura:

Prof. Dr.

Instituição: Assinatura:

Prof. Dr.

Instituição: Assinatura:

Prof. Dr.

Instituição: Assinatura:

Prof. Dr.

Instituição: Assinatura: 


\section{DEDICATÓRIA}

Por todas as noites em claro, dedico esse trabalho à minha mãe.

"Tudo aquilo que sou ou espero ser eu devo à minha mãe"

Abraham Lincoln 
Ao meu orientador, Luis Vicente Garcia, por ser a pessoa mais compreensiva que conheço, exemplo de vida e de princípios. Você me ajudou muito a ser uma pessoa melhor. E spero um dia poder agradecer por tudo.

"U m otimista vê uma oportunidade em cada calamidade. U m pessimista vê uma calamidade em cada oportunidade"

Winston Churchill 
A $D$ eus, 0 criador e o guia de todos nós. "E u sei que D eus não me dará nada com que eu não seja capaz de lidar. E u só gostaria que E le não confiasse tanto em mim"

Madre Teresa

Aos meus pais, José Antonio e Ramberlita, pela vida dedicada aos filhos, amor doado e princípios ensinados. V ocês são 0 alicerce sobre 0 qual ouso qualquer sonho. $M$ uito obrigada a vocês porque hoje encontro nas minhas conquistas as vossas presenças.

"Quando a raiz éfirme os ramos floresecem"

Ditado oriental

A minhas irmãs, Christiane e E liene, por sempre adicionarem o tempero necessário do encorajamento e do bom humor na minha vida.

"A s coisas que mais amamos nas pessoas se tornam mais claras na ausência delas" 
Ao meu esposo, $M$ atheus, que me deu carinho, coragem e conselhos. A cada dia crescemos juntos. Muito obrigada por permanecer ao meu lado.

"0 amor é a força mais abstrata e também a mais potente que há no mundo"

Gandhi

A os meus sogros, M auro e Carolina, por terem sido sempre tão carinhosos e compreensivos. M uito obrigada por tudo que já fizeram por mim!

"F ica sempre um pouco de perfume nas mãos que of erecem rosas"

Provérbio Chinês

A os funcionários do centro cirúrgico, em especial, Sérgio e os auxiliares da anestesia, pela paciência durante a fase de coleta dos dados. Obrigada por compreender 0 quanto isso era importante para mim.

"O que quer que você possa fazer ou sonhar que pode, comece.

A ousadia contém em si mesma genialidade, poder e magia" 
Às minhas melhores amigas, Ana Caroline, Renata e Ana Lúcia, por todas as horas compartilhadas, conselhos e sorrisos. Obrigada pela paciência.

"A ssim como os perfumes alegram a vida, a amizade sincera dá animo para viver"

Bíblia - Prov, 27-9

A todos que de alguma forma contribuíram para esse trabalho ou para minha formação pessoal ou profissional. Obrigada por tudo!

"O valor das coisas não está no tempo em que elas duram mas na intensidade com que acontecem. Por isso existem momentos inesquecíveis, coisas inexplicáveis e pessoas incomparáveis"

Fernando Pessoa 


\section{"A s pegadas na areia do tempo não são deixadas por quem fica sentado." Provérbio Berbere}




\section{RESUMO}

ARAUJO, L. M. T. Hemodiluição normovolêmica com solução hipertônica a 7,5\%. Parâmetros hemodinâmicos, efeitos metabólicos e repercussões laboratoriais. 2008. 186f. Tese (Doutorado) - Faculdade de Medicina, Universidade de São Paulo, Ribeirão Preto, 2008.

A hemodiluição normovolêmica aguda (HNA) é terapia mundialmente reconhecida como vantajosa em cirurgias que possuem grande potencial para sangramento, mas o edema provocado pela maior infusão de fluidos pode ser danoso em alguns pacientes. No intuito de avaliar as repercussões hemodinâmicas e laboratoriais da utilização da solução salina hipertônica a 7,5\% como líquido parcial de reposição na HNA foram estudados 20 pacientes submetidos à artrodese para correção de escoliose de coluna. No grupo 1 (SS 0,9\%, n=10) o sangue retirado na HNA, realizada momentos antes da cirurgia, foi reposto por solução salina $0,9 \%$ em um volume três vezes maior que o retirado. No grupo 2 (SS 7,5\%, $\mathrm{n}=10$ ) a metade do que foi retirado foi reposto com solução a $0,9 \%$ (três vezes o volume retirado) e a outra metade por $4 \mathrm{ml} \cdot \mathrm{kg}^{-1}$ de solução salina hipertônica 7,5\%. Nenhum dos pacientes apresentou distúrbios do equilíbrio ácido-básico e as alterações metabólicas (aumento da osmolaridade, sódio e cloro plasmáticos) foram passageiras. Os pacientes mantiveram-se hemodinamicamente estáveis e não foi observado sangramento anormal intra-operatório. A incidência de transfusão sangüínea foi semelhante embora os pacientes do grupo solução hipertônica tenham ficado clinicamente menos edemaciados. Mesmo não sendo objeto do estudo, observou-se menor incidência de infecções pós-operatórias nos pacientes submetidos à infusão de solução hipertônica, o que pode significar potencial efeito protetor da solução. Embora mais estudos com números maiores de pacientes sejam necessários para se comprovar esses efeitos, a solução hipertônica mostrou-se barata, simples e segura como maneira de redução do volume infundido na HNA.

Palavras-chave: Hemodiluição. Solução salina hipertônica. Escoliose. 


\section{A bstract}

PDF created with pdfFactory Pro trial version www.pdffactory.com 


\begin{abstract}
ARAUJO, L. M. T. Normovolemic hemodilution with $7.5 \%$ hypertonic solution. Hemodynamic parameters, metabolic effects and laboratorial repercussions. 2008. $186 f$. Tese (Doutorado) - Faculdade de Medicina, Universidade de São Paulo, Ribeirão Preto, 2008.

The acute normovolemic hemodilution (ANH) is a therapy recognized as benefic in surgeries that have a great potential for bleeding but the swelling caused by solutions infused intraoperative can be harmful in some kind of patients. Twenty patients submitted to arthrodesis for scoliosis correction were studied in order to evaluate the hemodynamic and laboratory effects of $7.5 \%$ hypertonic saline solution as a partial fluid replacement in ANH. In group 1 (SS $0.9 \%, \mathrm{n}=10$ ) the collected blood in ANH was replaced by saline solution $0.9 \%$ in a volume three times greater than the withdrawal. In group 2 (SS 7.5\%, $\mathrm{n}=10$ ), half of the blood removed was restored by $0.9 \%$ solution (three times the amount withdrawn) and the other half by $4 \mathrm{ml} . \mathrm{kg}^{-1}$ of $7.5 \%$ solution. None of the patients presented acid-base disorders. Metabolic changes - enhanced of plasmatic levels of sodium, chlorine and osmolarity - were all transitory. Patients remain hemodynamic stable and no abnormal bleeding was observed. They had similar incidence of blood transfusion but the ones in hypertonic solution group were clinically less swollen. Lower incidences of post-operative infections were found in this group, which could mean a possible protector potential of hypertonic solution. SS 7.5\% was a cheap, simple and safe alternative to reduce volume infused in ANH.
\end{abstract}

Keywords: Hemodilution. Hypertonic saline solution. Scoliosis. 


\section{LISTA DE GRÁFICOS}

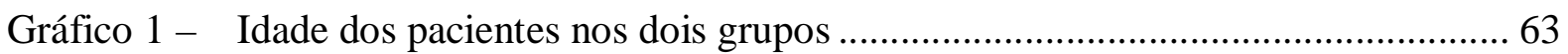

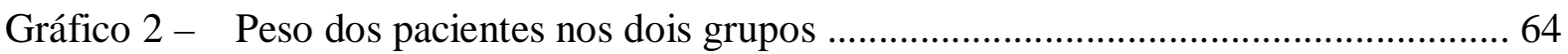

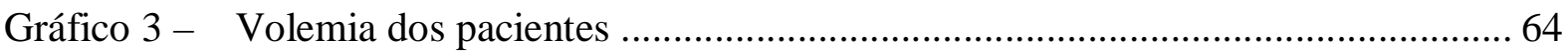

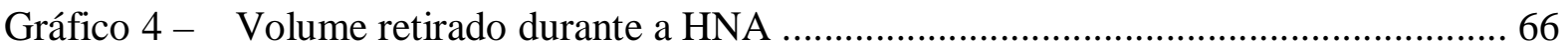

Gráfico 5 - Volume utilizado para reposição do que foi retirado durante a HNA ............. 66

Gráfico 6 - Tempo para coleta do volume de sangue durante a HNA …........................... 67

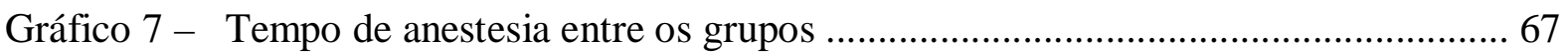

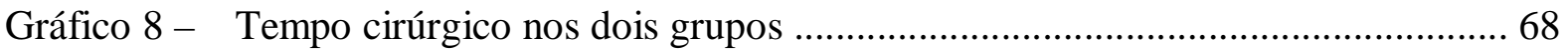

Gráfico 9 - Mediana, percentil 25 e percentil 75 do volume de sangue perdido por $\mathrm{Kg}$, durante o período intra-operatório, nos dois grupos ...........................68

Gráfico 10 - Volume total de cristalóides infundido em cada paciente no período intra-operatório, nos dois grupos

Gráfico 11 - Volume infundido por quilo de peso do paciente por hora de cirurgia, em cada grupo

Gráfico 12 - Diurese total de cada paciente no período intra-operatório, nos dois grupos.

Gráfico 13 - Diurese por quilo de peso por hora de cirurgia, em cada grupo 71

Gráfico 14 - Hemoglobina intra-operatória grupo a grupo, em cada tempo 72

Gráfico 15 - Hemoglobina intra-operatória tempo a tempo, em cada grupo 73

Gráfico 16 - Hematócrito intra-operatório grupo a grupo, em cada tempo 74

Gráfico 17 - Hematócrito intra-operatório tempo a tempo, em cada grupo .75

Gráfico 18 - Sódio plasmático intra-operatório grupo a grupo, em cada tempo 76

Gráfico 19 - Sódio plasmático intra-operatório tempo a tempo, em cada grupo 77 
Gráfico 20 - Potássio plasmático intra-operatório grupo a grupo, em cada tempo ............... 78

Gráfico 21 - Potássio plasmático intra-operatório tempo a tempo, em cada grupo ............... 79

Gráfico 22 - Cloro plasmático intra-operatório grupo a grupo, em cada tempo ................... 80

Gráfico 23 - Cloro plasmático intra-operatório tempo a tempo, em cada grupo .................. 81

Gráfico 24 - Cálcio plasmático intra-operatório grupo a grupo, em cada tempo ................. 82

Gráfico 25 - Cálcio plasmático intra-operatório tempo a tempo, em cada grupo .................. 83

Gráfico 26 - Lactato plasmático intra-operatório grupo a grupo, em cada tempo ................ 84

Gráfico 27 - Lactato plasmático intra-operatório tempo a tempo, em cada grupo ................. 85

Gráfico 28 - Glicemia plasmática intra-operatória grupo a grupo, em cada tempo .............. 86

Gráfico 29 - Glicemia plasmática intra-operatória tempo a tempo, em cada grupo ............. 87

Gráfico 30 - Osmolaridade plasmática intra-operatória grupo a grupo, em cada tempo ....... 88

Gráfico 31 - Osmolaridade plasmática intra-operatória tempo a tempo, em cada grupo ...... 89

Gráfico 32 - Frequiência cardíaca intra-operatória grupo a grupo, em cada tempo ............... 90

Gráfico 33 - Frequiência cardíaca intra-operatória tempo a tempo, em cada grupo...............91

Gráfico 34 - Pressão arterial sistólica intra-operatória grupo a grupo, em cada tempo ........ 92

Gráfico 35 - Pressão arterial diastólica intra-operatória grupo a grupo, em cada tempo ....... 93

Gráfico 36 - Pressão arterial média intra-operatória grupo a grupo, em cada tempo............ 94

Gráfico 37 - Pressão arterial intra-operatória tempo a tempo, em cada grupo ...................... 95

Gráfico 38 - Pressão venosa central intra-operatória grupo a grupo, em cada tempo .......... 96

Gráfico 39 - Pressão venosa central intra-operatória tempo a tempo, em cada grupo .......... 97

Gráfico 40 - Osmolaridade urinária intra-operatória grupo a grupo, em cada tempo ........... 98

Gráfico 41 - Osmolaridade urinária intra-operatória tempo a tempo, em cada grupo .......... 99

Gráfico 42 - Sódio urinário intra-operatório grupo a grupo, em cada tempo ..................... 100

Gráfico 43 - Sódio urinário intra-operatório tempo a tempo, em cada grupo ................... 101

Gráfico 44 - Potássio urinário intra-operatório grupo a grupo, em cada tempo ................. 102 
Gráfico 45 - Potássio urinário intra-operatório tempo a tempo, em cada grupo 103

Gráfico 46 - pH urinário intra-operatório tempo a tempo, em cada grupo 104

Gráfico 47 - pH urinário intra-operatório grupo a grupo, em cada tempo 105

Gráfico 48 - pH arterial nos dois grupos 106

Gráfico $49-\mathrm{pO}_{2}$ arterial nos dois grupos 107

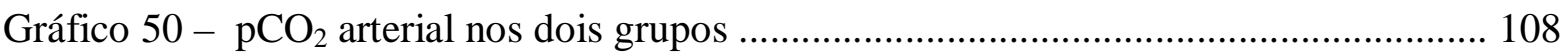

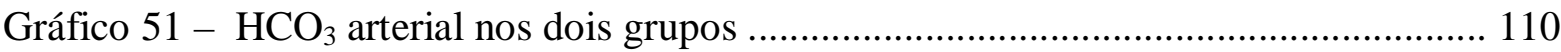

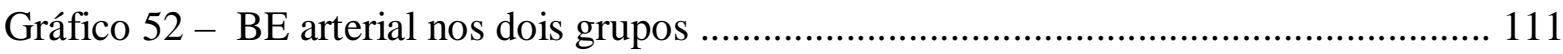

Gráfico 53 - Saturação arterial de oxigênio nos dois grupos .......................................... 112

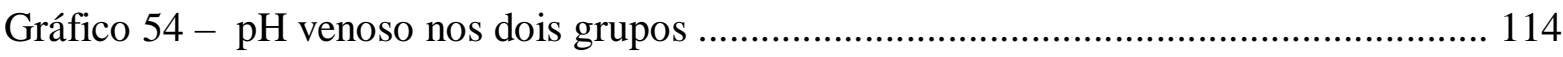

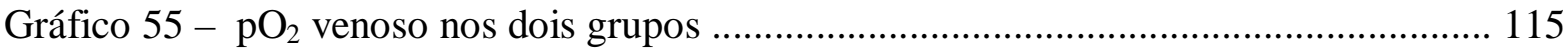

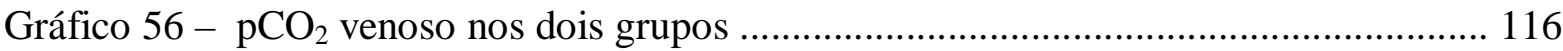

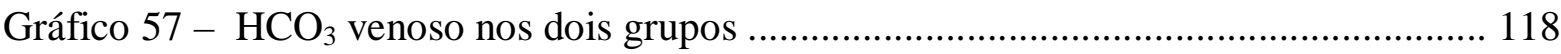

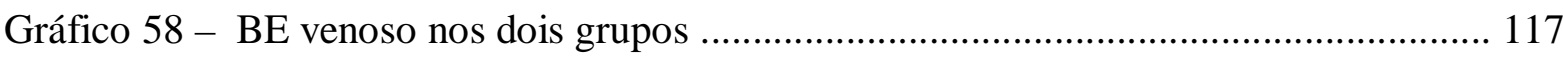

Gráfico 59 - Saturação venosa de oxigênio nos dois grupos .......................................... 118 


\section{LISTA DE FIGURAS}

Figura 1 - Monitorização habitual intra-operatória ....................................................... 53

Figura 2 - Aparelho utilizado para gravação dos dados hemodinâmicos ........................... 53

Figura 3 - Paciente em posição cirúrgica demonstrando o grau da escoliose ..................... 54

Figura 4 - Paciente anestesiado, pronto para o procedimento .......................................... 54

Figura 5 - Bomba para infusão contínua de propofol …................................................. 55

Figura 6 - Preparação da solução salina hipertônica ....................................................... 55

Figura 7 - Procedimento de pesagem das bolsas coletadas .............................................. 56

Figura 8 - Sangue retirado armazenado em bolsas durante a cirurgia .............................57

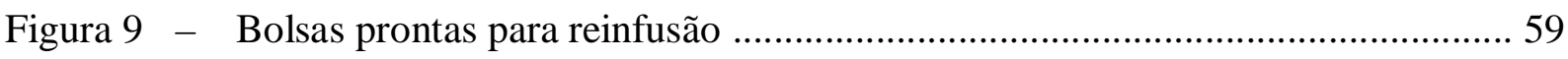

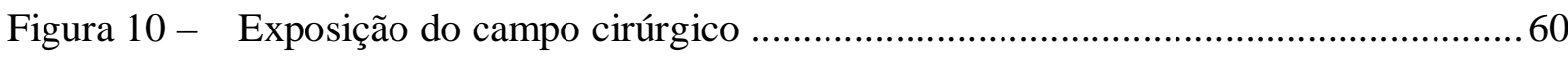




\section{LISTA DE TABELAS}

Tabela 1 - Dados demográficos . 65

Tabela 2 - Volume infundido por quilo de peso do paciente por hora de cirurgia em cada grupo 


\section{LISTA DE ABREVIATURAS E SIGLAS}

ACTH Hormônio adenocorticotrófico

ASA Classificação da Sociedade Americana de Anestesiologistas para o estado físico

AVA Artrodese por via anterior

AVP Artrodese por via posterior

BE $\quad$ Excesso de bases

$\mathrm{CaO}_{2} \quad$ Conteúdo arterial de oxigênio

CEC Circulação extra-corpórea

d Dalton

DAP Doação autóloga pré-operatória

DC Débito cardíaco

$\mathrm{DO}_{2} \quad$ Oferta de oxigênio

Dp Desvio padrão

$\mathrm{EtCO}_{2} \quad$ Volume expirado de gás carbônico

EUA Estados Unidos da América

FC Freqüência cardíaca

HIV Vírus da Imunodeficiência Humana

HNA Hemodiluição normovolêmica aguda

$\mathrm{pO}_{2} \quad$ Pressão de oxigênio

$\mathrm{pCO}_{2} \quad$ Pressão de gás carbônico

pH Potencial hidrogeniônico

PNA Peptídeo natriurético atrial 
PVC Pressão venosa central

RL Ringer com lactato

SS Solução salina

SSH Solução salina hipertônica

$\mathrm{SvO}_{2} \quad$ Saturação venosa de oxigênio

TAP Tempo de protrombina

$\mathrm{TEO}_{2} \quad$ Extração de oxigênio

TTPa Tempo de tromboplastina ativada

UTI Unidade de Terapia Intensiva

$\mathrm{VO}_{2} \quad$ Consumo de oxigênio 


\section{LISTA DE SÍMBOLOS}

$\begin{array}{ll}{ }^{\circ} \mathrm{C} & \text { Graus Celcius } \\ \mathrm{Cl} & \text { Cloro } \\ \mathrm{CO}_{2} & \text { Gás carbônico } \\ \mathrm{H} & \text { Hidrogênio } \\ \mathrm{HCO}_{3} & \text { Bicarbonato } \\ \mathrm{K} & \text { Potássio } \\ \mathrm{Na} & \text { Sódio } \\ \mathrm{NaCl} & \text { Cloreto de sódio } \\ \mathrm{NaCO}_{3} & \text { Bicarbonato de sódio } \\ \mathrm{O}_{2} & \text { Oxigênio }\end{array}$




\section{SUMÁRIO}

Folha de aprovação

Dedicatória

Agradecimento especial

Agradecimentos

Epígrafe

Resumo

Abstract

Lista de gráficos

Lista de figuras

Lista de tabelas

Lista de abreviaturas e siglas

Lista de símbolos

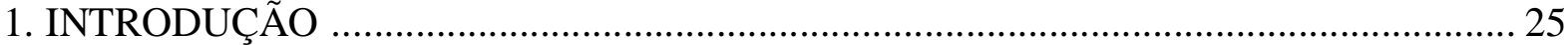

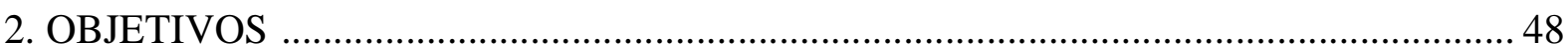

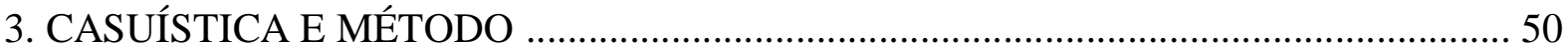

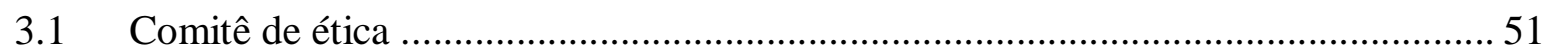

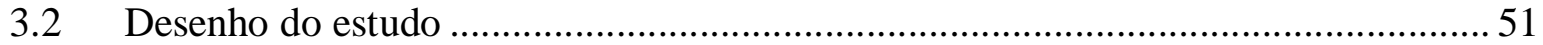

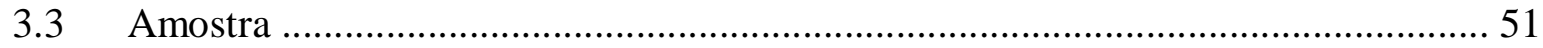

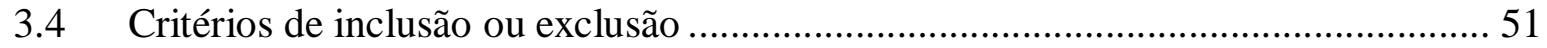

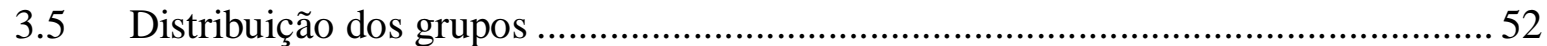

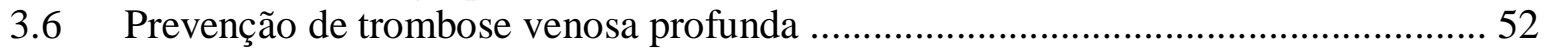

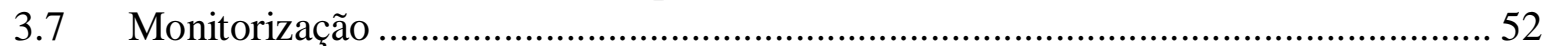

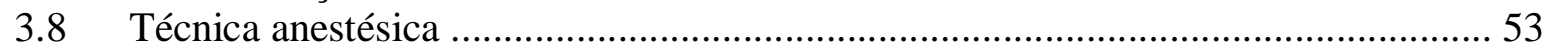

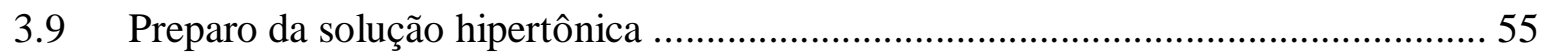

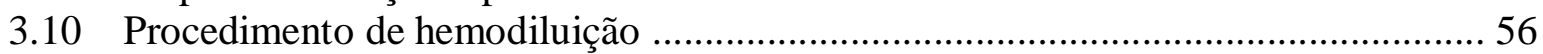

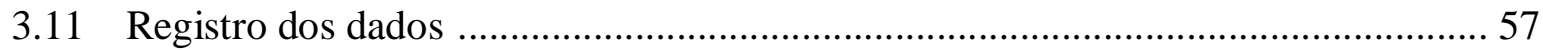

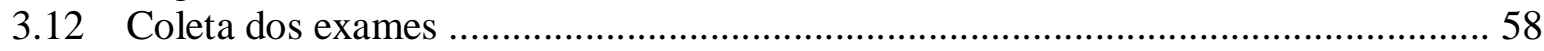

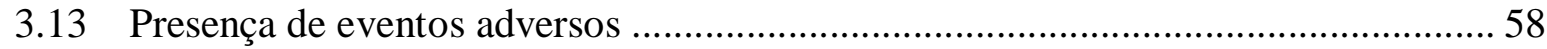


3.14 Critérios para reposição do volume da hemodiluição ...........................................59

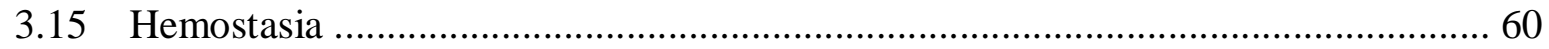

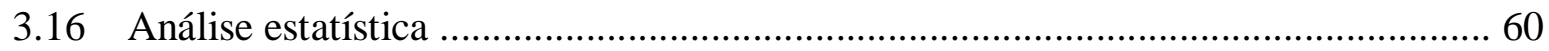

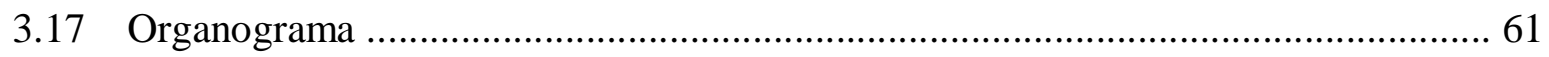

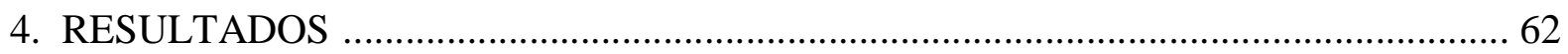

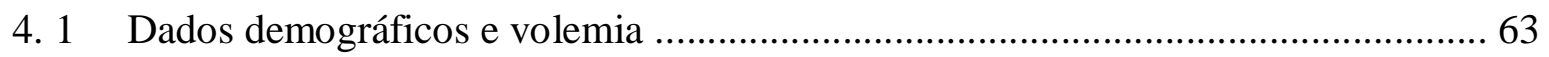

4. 2 Realização da HNA e dados gerais do período intra-operatório ............................... 65

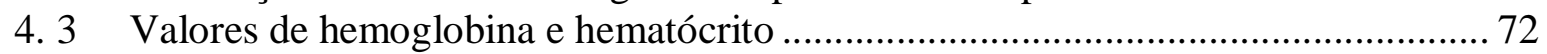

4. 4 Efeitos metabólicos e laboratoriais plasmáticos .................................................. 75

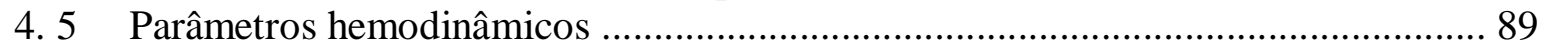

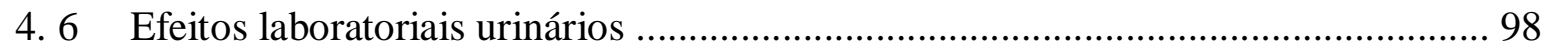

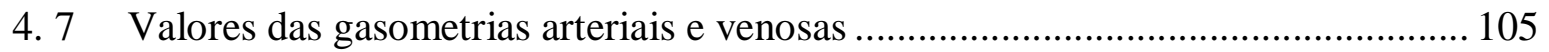

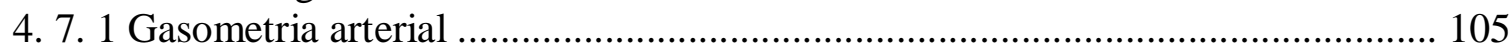

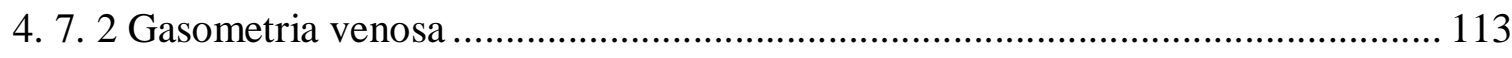

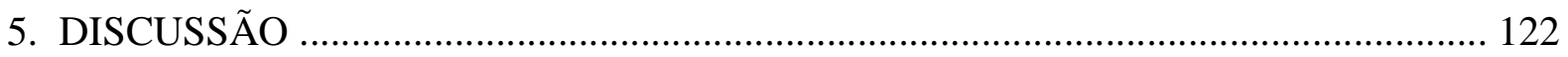

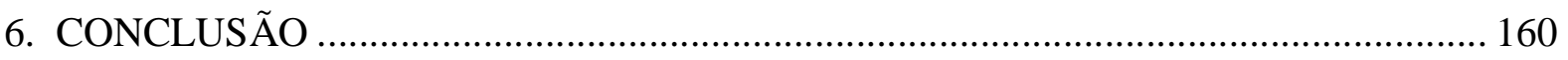

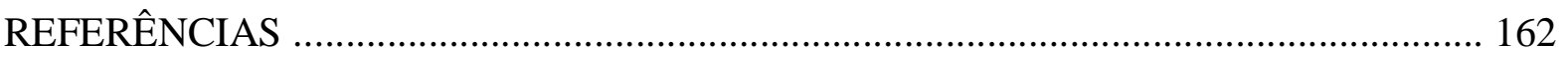

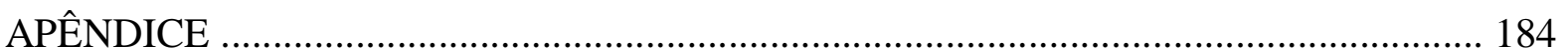

Apêndice 1 - Termo de consentimento livre e esclarecido ............................................... 185 